\title{
A Study of Bilateral New Mock Theta Functions
}

\author{
Bhaskar Srivastava
}

Department of Mathematics and Astronomy, Lucknow University, Lucknow, India

\begin{abstract}
We define bilateral series for two sets of new mock theta functions-- one given by Andrews and the other by Bringmann et al. Not only the bilateral form of the mock theta functions in the two sets are equivalent they further come out to be equivalent to the bilateral form of the eighth order mock theta functions of Gordon and McIntosh. These bilateral series are expressed as a $q$-hypergeometric ${ }_{2} \varphi_{1}$ series and then represented by continued fractions. We extend the definition of the bilateral mock theta functions and show they are $F_{q}$-functions and relations are then defined.
\end{abstract}

Keywords Mock Theta Functions, Bilateral Series, Continued Fractions

\section{Introduction}

In his last letter to G.H. Hardy[11], S. Ramanujan listed seventeen mock theta functions of order three, five, five and seven. According to Ramanujan a mock theta function is a function $f(q),|q|<1$, satisfying the following two conditions:

(0) For every root of unity $\zeta$, there is a $\theta$-function $\theta_{\zeta}(q)$ such that the difference $f(q)-\theta_{\zeta}(q)$ is bounded as $q \rightarrow \zeta$ radially.

(1) There is no single $\theta$-function which works for all $\zeta$ i.e., for every $\theta$-function $\theta(q)$ there is some root of unity $\zeta$ for which $f(q)-\theta(q)$ is unbounded as $q \rightarrow \zeta$ radially.

G.N. Watson[17] found three more mock theta functions of order three. In his "Lost" Notebook Ramanujan gave six more mock theta functions which were called by G.E. Andrews and D. Hickerson[5] of order six and four mock theta functions which were called by Choi[6] of order ten. B. Gordon and R.J. McIntosh[8] generated eight mock theta functions and called them of order eight, but four of them were later found of lower order. Hikami[9] found one more mock theta function of order two.

Recently Andrews[2] in his paper generated some new mock theta functions and found four of them interesting. Bringmann, Hikami and Lovejoy developed two more mock theta functions. We in[12-14] have made a comprehensive study of these mock theta functions.

After studying these mock theta functions in[12-14] I started considering their bilateral form. The study became interesting as in their bilateral form the mock theta functions

* Corresponding author:

bhaskarsrivastav@yahoo.com (Bhaskar Srivastava)

Published online at http://journal.sapub.org/ajms

Copyright (C) 2012 Scientific \& Academic Publishing. All Rights Reserved $\bar{\psi}_{0}(q)$ and $\bar{\psi}_{1}(q)$ of Andrews came out equivalent to the bilateral form of mock theta functions $\bar{\phi}_{0}(q)$ and $\bar{\phi}_{1}(q)$ of Bringmann et al. Moreover the bilateral $\bar{\psi}_{0, c}(q)$ and $\bar{\psi}_{1, c}(q)$ are found to be equivalent to bilateral form of the eighth order mock theta functions $S_{0}(q)$ and $S_{1}(q)$, generated by Gordon and McIntosh. Also $\bar{\phi}_{0, c}(q)$ and $\bar{\phi}_{1, c}(q)$ are equivalent to the bilateral form of the mock theta functions $T_{0}(q)$ and $T_{1}(q)$ of Gordon and McIntosh. Lastly I represent these bilateral form of the mock theta functions as $q$-hypergeometric ${ }_{2} \phi_{1}$ series and write them as continued fractions.

The four mock theta functions of Andrews [2]:

$$
\begin{aligned}
\bar{\psi}_{0}(q) & =\sum_{n=0}^{\infty} \frac{q^{2 n^{2}}}{(-q ; q)_{2 n}}, \\
\bar{\psi}_{1}(q) & =\sum_{n=0}^{\infty} \frac{q^{2 n^{2}+2 n}}{(-q ; q)_{2 n+1}}, \\
\bar{\psi}_{2}(q) & =\sum_{n=0}^{\infty} \frac{q^{2 n^{2}+2 n}\left(q ; q^{2}\right)_{n}}{\left(q^{2} ; q^{2}\right)_{n}(-q ; q)_{2 n}},
\end{aligned}
$$

and

$$
\bar{\psi}_{3}(q)=\sum_{n=0}^{\infty} \frac{q^{n^{2}}(-q ; q)_{n}^{2}}{(q ; q)_{2 n}} .
$$

Andrews called $\bar{\psi}_{3}(q)$ as a companion to the third order mock theta function of Ramanujan

$$
\psi(q)=\sum_{n=0}^{\infty} \frac{q^{n^{2}}}{\left(q ; q^{2}\right)_{n}} .
$$

The two mock theta functions of Bringmann, Hikami and Lovejoy [10]:

$$
\bar{\phi}_{0}(q)=\sum_{n=0}^{\infty} q^{n}(-q ; q)_{2 n+1}
$$

and 


$$
\overline{\phi_{1}}(q)=\sum_{n=0}^{\infty} q^{n}(-q ; q)_{2 n} .
$$

We now write the mock theta function as basic bilateral series, and following Watson, call them 'Complete'.

$$
\begin{aligned}
& \bar{\psi}_{0, c}(q)=\sum_{n=-\infty}^{\infty} \frac{q^{2 n^{2}}}{(-q ; q)_{2 n}}, \\
& \bar{\psi}_{1, c}(q)=\sum_{n=-\infty}^{\infty} \frac{q^{2 n^{2}+2 n}}{(-q ; q)_{2 n+1}}, \\
& \bar{\phi}_{0, c}(q)=\sum_{n=-\infty}^{\infty} q^{n}(-q ; q)_{2 n+1},
\end{aligned}
$$

and

$$
\bar{\phi}_{1, c}(q)=\sum_{n=-\infty}^{\infty} q^{n}(-q ; q)_{2 n} .
$$

The scheme of the paper is as follows:

In section 3, we give an expansion for these functions using Slater's expansion formula[7] and show their relationship with other bilateral mock theta functions.

In section 4 and section 5, we express these 'complete' mock theta functions as ${ }_{2} \varphi_{1}$ series.

In section 6, we represent these 'complete' mock theta functions as continued fractions.

In section 7, we give alternative definitions, using Bailey's bilateral transformation[7].

In section 8 , relationship between two sets of bilateral mock theta functions is shown.

In section 9 and 10, we define extended form for the 'complete' mock theta functions and show that they are $F_{q}$ functions and certain relationship between these functions.

\section{Basic Standard Results}

We shall use the following usual basic hypergeometric notations:

$$
\left(a ; q^{k}\right)_{n}=\prod_{m=1}^{n}\left(1-a q^{k(m-1)}\right), \quad\left|q^{k}\right|<1, n, \text { non negative inte- }
$$

ger

$$
\begin{gathered}
\left(a ; q^{k}\right)_{0}=1, \\
\left(a ; q^{k}\right)_{\infty}=\prod_{m=1}^{\infty}\left(1-a q^{k(m-1)}\right) \\
\left(a_{1}, a_{2}, \ldots \ldots, a_{m} ; q^{k}\right)_{n}=\left(a_{1} ; q^{k}\right)_{n}\left(a_{2} ; q^{k}\right)_{n} \ldots \ldots\left(a_{m} ; q^{k}\right)_{n} . \\
{ }_{A} \varphi_{A-1}\left[a_{1}, a_{2}, \ldots, a_{A} ; b_{1}, b_{2} \ldots, b_{A-1} ; q_{1}, z\right] \\
=\sum_{n=0}^{\infty} \frac{\left(a_{1} ; q_{1}\right)_{n} \ldots .\left(a_{A} ; q_{1}\right)_{n} z^{n}}{\left(b_{1} ; q_{1}\right)_{n} \ldots .\left(b_{A-1} ; q_{1}\right)_{n}\left(q_{1} ; q_{1}\right)_{n}},|z|<1 .
\end{gathered}
$$

A generalized basic bilateral series is defined as

$$
{ }_{r} \psi_{r}\left[\begin{array}{l}
a_{1}, \ldots . ., a_{r} \\
b_{1}, \ldots . ., b_{r}
\end{array} ; q ; z\right]=\sum_{n=-\infty}^{\infty} \frac{\left(a_{1}, a_{2}, \ldots \ldots ., a_{r} ; q\right)_{n}}{\left(b_{1}, b_{2}, \ldots \ldots, b_{r} ; q\right)_{n}} z^{n},
$$

where $\left|b_{1} b_{2} \ldots \ldots b_{r} / a_{1} a_{2} \ldots \ldots a_{r}\right|<|z|<1$.

\section{Relations between Bilateral Mock Theta Functions}

Taking $r=2$ in the following expansion of Slater [7, (5.4.3), p. 129]:

$$
\begin{aligned}
& \frac{\left(b_{1}, b_{2}, q / a_{1}, q / a_{2}, d z, q / d z ; q\right)_{\infty}}{\left(c_{1}, c_{2}, q / c_{1}, q / c_{2} ; q\right)_{\infty}}{ }_{2} \psi_{2}\left[\begin{array}{l}
a_{1}, a_{2} \\
b_{1}, b_{2}
\end{array} ;, z\right] \\
& =\frac{q}{c_{1}} \frac{\left(c_{1} / a_{1}, c_{1} / a_{2}, q b_{1} / c_{1}, q b_{2} / c_{1}, d c_{1} z / q, q^{2} / d c_{1} z ; q\right)_{\infty}}{\left(c_{1}, q / c_{1}, c_{1} / c_{2}, q c_{2} / c_{1} ; q\right)_{\infty}} \\
& { }_{2} \psi_{2}\left[\begin{array}{l}
q a_{1} / c_{1}, q a_{2} / c_{1} \\
q b_{1} / c_{1}, q b_{2} / c_{1}
\end{array} ; q, z\right]+\operatorname{idem}\left(c_{1} ; c_{2}\right),
\end{aligned}
$$

where $d=a_{1} a_{2} / c_{1} c_{2},\left|b_{1} b_{2} / a_{1} a_{2}\right|<|z|<1$, and idem $\left(c_{1} ; c_{2}\right)$ after the expression means that the preceding expression is repeated with $c_{1}$ and $c_{2}$ interchanged.

We now give expansions for these bilateral mock theta functions.

(i) Letting $q \rightarrow q^{2}, a_{1}, a_{2} \rightarrow \infty$ and taking $b_{1}=-q$, $b_{2}=-q^{2}, \quad z=q^{2} / a_{1} a_{2}$ in (3.1), we have

$$
\begin{aligned}
& \frac{\left(-q,-q^{2}, q^{2} / c_{1} c_{2}, c_{1} c_{2} ; q^{2}\right)_{\infty}}{\left(c_{1}, c_{2}, q^{2} / c_{1}, q^{2} / c_{2} ; q^{2}\right)_{\infty}} \bar{\psi}_{0, c}(q) \\
&= \frac{q^{2}}{c_{1}} \frac{\left(-q^{3} / c_{1},-q^{4} / c_{1}, 1 / c_{2}, q^{2} c_{2} ; q^{2}\right)_{\infty}}{\left(c_{1}, q^{2} / c_{1}, c_{1} / c_{2}, q^{2} c_{2} / c_{1} ; q^{2}\right)_{\infty}} \\
& \sum_{n=-\infty}^{\infty} \frac{q^{2 n^{2}+4 n}}{\left(-q^{3} / c_{1} ; q^{2}\right)_{n}\left(-q^{4} / c_{1} ; q^{2}\right)_{n} c_{1}^{2 n}}+\operatorname{idem}\left(c_{1} ; c_{2}\right) . \\
&\text { (ii) } \left.\frac{(1+q)\left(-q^{2},-q^{3}, q^{4} / c_{1} c_{2}, c_{1} c_{2} / q^{2} ; q^{2}\right)_{\infty}}{{ }_{1}, c_{1, c}(q)} q^{2} / c_{1}, q^{2} / c_{2} ; q^{2}\right)_{\infty} \\
&=\frac{q^{2}}{c_{1}} \frac{\left(-q^{4} / c_{1},-q^{5} / c_{1}, q^{2} / c_{2}, c_{2} ; q^{2}\right)_{\infty}}{\left(c_{1}, q^{2} / c_{1}, c_{1} / c_{2}, q^{2} c_{2} / c_{1} ; q^{2}\right)_{\infty}} \\
& \sum_{n=-\infty}^{\infty} \frac{q^{2 n^{2}+6 n}}{\left(-q^{4} / c_{1} ; q^{2}\right)_{n}\left(-q^{5} / c_{1} ; q^{2}\right)_{n} c_{1}^{2 n}} \\
& \quad+\operatorname{idem}\left(c_{1} ; c_{2}\right) .
\end{aligned}
$$$$
\left(q \rightarrow q^{2}, a_{1}, a_{2} \rightarrow \infty, b_{1}=-q^{2}, \quad b_{2}=-q^{3}, \quad z=q^{4} / a_{1} a_{2}\right. \text { in }
$$

$$
\text { (iii) } \begin{aligned}
\frac{2\left(-q,-q^{2}, q^{6} / c_{1} c_{2}, c_{1} c_{2} / q^{4} ; q^{2}\right)_{\infty}}{q\left(c_{1}, c_{2}, q^{2} / c_{1}, q^{2} / c_{2} ; q^{2}\right)_{\infty}} \bar{\phi}_{0, c}(q) \\
=\frac{q^{2}}{c_{1}} \frac{\left(-c_{1} / q^{2},-c_{1} / q^{3}, q^{4} / c_{2}, c_{2} / q^{2} ; q^{2}\right)_{\infty}}{\left(c_{1}, q^{2} / c_{1}, c_{1} / c_{2}, q^{2} c_{2} / c_{1} ; q^{2}\right)_{\infty}} \\
\sum_{n=-\infty}^{\infty} q^{n}\left(-q^{4} / c_{1} ; q^{2}\right)_{n}\left(-q^{5} / c_{1} ; q^{2}\right)_{n} \\
\quad+\operatorname{idem}\left(c_{1} ; c_{2}\right) .
\end{aligned}
$$

$\left(q \rightarrow q^{2}, \quad a_{1}=-q^{2}, a_{2}=-q^{3}, \quad b_{1}=b_{2}=0, z=q\right.$ in (3.1))

$$
\text { (iv) } \begin{aligned}
& \frac{\left(-q,-1, q^{4} / c_{1} c_{2}, c_{1} c_{2} / q^{2} ; q^{2}\right)_{\infty}}{\left(c_{1}, c_{2}, q^{2} / c_{1}, q^{2} / c_{2} ; q^{2}\right)_{\infty}} \bar{\phi}_{1, c}(q) \\
= & \frac{q^{2}}{c_{1}} \frac{\left(-c_{1} / q,-c_{1} / q^{2}, q^{2} / c_{2}, c_{2} ; q^{2}\right)_{\infty}}{\left(c_{1}, q^{2} / c_{1}, c_{1} / c_{2}, q^{2} c_{2} / c_{1} ; q^{2}\right)_{\infty}}
\end{aligned}
$$




$$
\begin{aligned}
& \sum_{n=-\infty}^{\infty} q^{n}\left(-q^{3} / c_{1} ; q^{2}\right)_{n}\left(-q^{4} / c_{1} ; q^{2}\right)_{n} \\
& \quad+\operatorname{idem}\left(c_{1} ; c_{2}\right) .
\end{aligned}
$$$$
\left(q \rightarrow q^{2}, \quad a_{1}=-q, \quad a_{2}=-q^{2}, \quad b_{1}=b_{2}=0, \quad z=q\right. \text { in (3.1)) }
$$

Relations between bilateral mock theta functions Special Cases

Take $c_{1}=q$ in (3.2):

$$
\begin{aligned}
\bar{\psi}_{0, c}(q)= & -\frac{q\left(1-c_{2}\right)^{2}}{(1+q) c_{2}} \frac{\left(q^{2} c_{2}, q^{2} c_{2}, q^{2} / c_{2}, q^{2} / c_{2} ; q^{2}\right)_{\infty}}{\left(q c_{2}, q c_{2}, q / c_{2}, q / c_{2} ; q^{2}\right)_{\infty}} \bar{\psi}_{1, c}(q) \\
& +\operatorname{idem~}\left(q ; c_{2}\right) .
\end{aligned}
$$

Take $c_{1}=q^{3}$ in (3.3):

$$
\begin{aligned}
\bar{\psi}_{1, c}(q)= & -\frac{1}{c_{2}} \frac{\left(c_{2}, c_{2}, q^{2} / c_{2}, q^{2} / c_{2} ; q^{2}\right)_{\infty}}{\left(q c_{2}, q c_{2}, q / c_{2}, q / c_{2} ; q^{2}\right)_{\infty}} \bar{\psi}_{0, \mathrm{c}}(q) \\
& +\operatorname{idem~}\left(q^{3} ; c_{2}\right) .
\end{aligned}
$$

Take $c_{1}=q^{3}$ in (3.4):

$$
\begin{aligned}
\bar{\phi}_{0, c}(q)= & -\frac{c_{2}}{q^{2}} \frac{\left(c_{2}, c_{2}, q^{2} / c_{2}, q^{2} / c_{2} ; q^{2}\right)_{\infty}}{\left(c_{2} / q, c_{2} / q, q^{3} / c_{2}, q^{3} / c_{2} ; q^{2}\right)_{\infty}} \bar{\phi}_{1, \mathrm{c}}(q) \\
& +\operatorname{idem}\left(q^{3} ; c_{2}\right) .
\end{aligned}
$$

Take $c_{1}=q$ in (3.5):

$$
\begin{aligned}
\bar{\phi}_{1, c}(q)=- & \frac{q}{c_{2}} \frac{\left(c_{2}, c_{2}, q^{2} / c_{2}, q^{2} / c_{2} ; q^{2}\right)_{\infty}}{\left(q c_{2}, q c_{2}, q / c_{2}, q / c_{2} ; q^{2}\right)_{\infty}} \bar{\phi}_{0, \mathrm{c}}(q) \\
& +\operatorname{idem}\left(q ; c_{2}\right)
\end{aligned}
$$

\section{Expansion of Bilateral Mock Theta Functions $\bar{\psi}_{0, c}(q)$ and $\bar{\psi}_{1, c}(q)$ as ${ }_{2} \varphi_{1}$ Series}

In (3.1) we take $c_{j}=b_{j}, j=1,2$ to get [7, (5.4.5), p. 130]

$$
\begin{aligned}
& \quad \frac{\left(q / a_{1}, q / a_{2}, d z, q / d z ; q\right)_{\infty}}{\left(q / b_{1}, q / b_{2} ; q\right)_{\infty}}{ }_{2} \psi_{2}\left[\begin{array}{l}
a_{1}, a_{2} \\
b_{1}, b_{2} ; q, z
\end{array}\right] \\
& =\frac{q}{b_{1}} \frac{\left(q, b_{1} / a_{1}, b_{1} / a_{2}, d b_{1} z / q, q^{2} / d b_{1} z ; q\right)_{\infty}}{\left(b_{1}, q / b_{1}, b_{1} / b_{2} ; q\right)_{\infty}}{ }_{2} \varphi_{1}\left[\begin{array}{l}
q a_{1} / b_{1}, q a_{2} / b_{1} \\
q b_{2} / b_{1}
\end{array} ;, z\right] \\
& \quad+\operatorname{idem~}\left(b_{1} ; b_{2}\right),
\end{aligned}
$$

where $d=a_{1} a_{2} / b_{1} b_{2}$.

We will now specialize the paramaeter in (4.1) to get expansions of bilateral mock theta functions as $q$ - hypergeometric series.

(i) Letting $q \rightarrow q^{2}, \quad a_{1}, a_{2} \rightarrow \infty, \quad b_{1}=-q, \quad b_{2}=-q^{2} \quad$ and $z=q^{2} / a_{1} a_{2}$ in (4.1), to get

$$
\begin{aligned}
\bar{\psi}_{0, c}(q)= & -\frac{4 q}{1-q} \frac{\left(q^{2},-q^{2},-q^{2},-q^{2} ; q^{2}\right)_{\infty}}{\left(-q, q, q, q ; q^{2}\right)_{\infty}} \sum_{n=0}^{\infty} \frac{q^{2 n^{2}+2 n}}{\left(q^{2} ; q^{2}\right)_{n}\left(q^{3} ; q^{2}\right)_{n}} \\
& +\operatorname{idem}\left(b_{1} ; b_{2}\right) .
\end{aligned}
$$

(ii) $\bar{\psi}_{1, c}(q)=\frac{4(1+q)\left(q^{2},-q^{2},-q^{2},-q^{2} ; q^{2}\right)_{\infty}}{\left(-q, q, q, q ; q^{2}\right)_{\infty}} \sum_{n=0}^{\infty} \frac{q^{2 n^{2}}}{\left(q ; q^{2}\right)_{n}\left(q^{2} ; q^{2}\right)_{n}}$

$$
+ \text { idem }\left(b_{1} ; b_{2}\right) \text {. }
$$

$\left(q \rightarrow q^{2}, a_{1}, a_{2} \rightarrow \infty, \quad b_{1}=-q^{3}, b_{2}=-q^{2}\right.$ and $z=q^{4} / a_{1} a_{2}$ in (4.1))

\section{Expansions of Bilateral Mock Theta Functions $\bar{\phi}_{0, c}(q)$ and $\bar{\phi}_{1, c}(q)$ as ${ }_{2} \varphi_{1}$ Series}

For getting the expansion of a bilateral series as a ${ }_{2} \varphi_{1}$ series, we shall use the following expansion formula, which is obtained by putting $c_{j}=q a_{j}, j=1,2$ in Slater's expansion formula (3.1) [7, (5.4.4), p. 130]:

$$
\begin{aligned}
& \frac{\left(b_{1}, b_{2}, q / a_{1}, q / a_{2}, z, q / z ; q\right)_{\infty}}{\left(q a_{1}, q a_{2}, 1 / a_{1}, 1 / a_{2} ; q\right)_{\infty}}{ }_{2} \psi_{2}\left[\begin{array}{l}
a_{1}, a_{2} \\
b_{1}, b_{2}
\end{array} ;, z\right] \\
& =\frac{a_{1}\left(q, q a_{1} / a_{2}, b_{1} / a_{1}, b_{2} / a_{1}, a_{1} z, q / a_{1} z ; q\right)_{\infty}}{\left(q a_{1}, 1 / a_{1}, a_{1} / a_{2}, q a_{2} / a_{1} ; q\right)_{\infty}}{ }_{2} \phi_{1}\left[\begin{array}{c}
q a_{1} / b_{1}, q a_{1} / b_{2} \\
q a_{1} / a_{2}
\end{array} ;, b_{1} b_{2} / a_{1} a_{2} z\right] \\
& + \text { idem }\left(a_{1} ; a_{2}\right) \text {, }
\end{aligned}
$$

where $\left|b_{1} b_{2} / a_{1} a_{2}\right|<|z|<1$.

(i) Letting $q \rightarrow q^{2}, a_{1}=-q^{2}, \quad a_{2}=-q^{3}, b_{1}=b_{2}=0$ and $z=q$ in (5.1), we have

$$
\begin{aligned}
\bar{\phi}_{0, c}(q)= & \frac{\left(q^{2},-q,-q,-q ; q^{2}\right)_{\infty}}{2 q\left(-q^{2}, q, q, q ; q^{2}\right)_{\infty}} \sum_{n=0}^{\infty} \frac{q^{2 n^{2}}}{(q ; q)_{2 n}} \\
& +\operatorname{idem~}\left(a_{1} ; a_{2}\right) .
\end{aligned}
$$
(ii) $\bar{\phi}_{1, c}(q)=-\frac{1}{2(1-q)} \frac{\left(q^{2},-q,-q,-q ; q^{2}\right)_{\infty}}{\left(-q^{2}, q, q, q, q^{2}\right)_{\infty}} \sum_{n=0}^{\infty} \frac{q^{2 n^{2}+2 n}}{\left(q^{2} ; q\right)_{2 n}}$ + idem $\left(a_{1} ; a_{2}\right)$.

$\left(q \rightarrow q^{2}, \quad a_{1}=-q^{2}, \quad a_{2}=-q, \quad b_{1}=b_{2}=0 \quad\right.$ and $\quad z=q \quad$ in (5.1))

\section{Bilateral or 'Complete' Mock Theta Functions as Continued Fractions}

We first write the bilateral $\bar{\psi}_{0, c}(q)$ as the sum of two ${ }_{2} \phi_{2}$ basic hypergeometric series and then use the continued fraction [1,(3.79) p 82]:

$$
\frac{\sum_{n=0}^{\infty} \frac{q^{n^{2}} \lambda^{n}}{(q)_{n}(-\beta)_{n}}}{\sum_{n=0}^{\infty} \frac{q^{n^{2}+n} \lambda^{n}}{(q)_{n}(-\beta)_{n+1}}}=1+\frac{\lambda q+\beta}{1-\beta} \frac{\lambda q^{2}+\beta}{1-\beta+\ldots .},
$$

to represent $\bar{\psi}_{0, c}(q)$ as a continued fraction.

(i) Now (4.2) gives

$$
\begin{aligned}
\bar{\psi}_{0, c}(q)= & -\frac{4 q}{1-q} \frac{\left(q^{2},-q^{2},-q^{2},-q^{2} ; q^{2}\right)_{\infty}}{\left(-q, q, q, q ; q^{2}\right)_{\infty}} \sum_{n=0}^{\infty} \frac{q^{2 n^{2}+2 n}}{\left(q^{2} ; q^{2}\right)_{n}\left(q^{3} ; q^{2}\right)_{n}} \\
& +\frac{\left(q^{2},-q,-q,-q ; q^{2}\right)_{\infty}}{\left(-q^{2}, q, q, q ; q^{2}\right)_{\infty}} \sum_{n=0}^{\infty} \frac{q^{2 n^{2}}}{\left(q^{2} ; q^{2}\right)_{n}\left(q ; q^{2}\right)_{n}}
\end{aligned}
$$




$$
=S \sum_{n=0}^{\infty} \frac{q^{2 n^{2}+2 n}}{\left(q^{2} ; q^{2}\right)_{n}\left(q^{3} ; q^{2}\right)_{n}}+T \sum_{n=0}^{\infty} \frac{q^{2 n^{2}}}{\left(q^{2} ; q^{2}\right)_{n}\left(q ; q^{2}\right)_{n}}
$$

where

$$
S=-\frac{4 q}{1-q} \frac{\left(q^{2},-q^{2},-q^{2},-q^{2} ; q^{2}\right)_{\infty}}{\left(-q, q, q, q ; q^{2}\right)_{\infty}}
$$

and

$$
T=\frac{\left(q^{2},-q,-q,-q ; q^{2}\right)_{\infty}}{\left(-q^{2}, q, q, q ; q^{2}\right)_{\infty}} .
$$

Dividing the expressions on both sides of (6.2) by the first summation on the right side of (6.2), we have

$$
\frac{\bar{\psi}_{0, \mathrm{c}}(q)}{\sum_{n=0}^{\infty} \frac{q^{2 n^{2}+2 n}}{\left(q^{2} ; q^{2}\right)_{n}\left(q^{3} ; q^{2}\right)_{n}}}=S+T \frac{\sum_{n=0}^{\infty} \frac{q^{2 n^{2}}}{\left(q^{2} ; q^{2}\right)_{n}\left(q ; q^{2}\right)_{n}}}{\sum_{n=0}^{\infty} \frac{q^{2 n^{2}+2 n}}{\left(q^{2} ; q^{2}\right)_{n}\left(q^{3} ; q^{2}\right)_{n}}}
$$

Letting $q \rightarrow q^{2}, \lambda=1, \quad \beta=-q$ in (6.1), and then putting it in the quotient of the summation on the right side of (6.3) to have

$$
\frac{(1+q) \bar{\psi}_{0, \mathrm{c}}(q)}{\sum_{n=0}^{\infty} \frac{q^{2 n^{2}+2 n}}{\left(q^{2} ; q^{2}\right)_{n}\left(q^{3} ; q^{2}\right)_{n}}}=S+T\left[1+\frac{q^{2}-q}{(1+q)} \frac{q^{4}-q}{(1+q)+\ldots . .}\right]
$$

(ii) Similarly by (4.3), we have

$$
\bar{\psi}_{1, c}(q)=S_{1} \sum_{n=0}^{\infty} \frac{q^{2 n^{2}}}{\left(q ; q^{2}\right)_{n}\left(q^{2} ; q^{2}\right)_{n}}+T_{1} \sum_{n=0}^{\infty} \frac{q^{2 n^{2}+2 n}}{\left(q^{3} ; q^{2}\right)_{n}\left(q^{2} ; q^{2}\right)_{n}}
$$

where

$$
S_{1}=\frac{4\left(q^{2},-q^{2},-q^{2},-q^{2} ; q^{2}\right)_{\infty}}{\left(-q, q, q, q ; q^{2}\right)_{\infty}}
$$

and

$$
T_{1}=-\frac{\left(q^{2},-q,-q,-q ; q^{2}\right)_{\infty}}{(1-q)\left(-q^{2}, q, q, q ; q^{2}\right)_{\infty}} .
$$

Hence we have

$$
\frac{\bar{\psi}_{l c}(q)}{\sum_{n=0}^{\infty} \frac{q^{2 n^{2}+2 n}}{\left(q^{3} ; q^{2}\right)_{n}\left(q^{2} ; q^{2}\right)_{n}}}=T_{1}+S_{1}\left[1+\frac{q^{2}-q}{(1+q)} \frac{q^{4}-q}{(1+q)+\ldots . .}\right]
$$

(iii) For $\bar{\phi}_{0, c}(q)$ we will have the same continued fraction by (8.2).

(iv) For $\bar{\phi}_{1, c}(q)$ we will have the same continued fraction by (8.4).

\section{Alternative Definitions for 'Complete' Mock Theta Functions}

We shall use the following bilateral transformation of Bailey [7, 5.20(ii), p 137] to get alternative definition :

$$
{ }_{2} \psi_{2}\left[\begin{array}{l}
a, b \\
c, d
\end{array} ; q, z\right]=\frac{(a z, d / a, c / b, d q / a b z ; q)_{\infty}}{(z, d, q / b, c d / a b z ; q)_{\infty}} \times
$$

$$
\times{ }_{2} \psi_{2}\left[\begin{array}{c}
a, a b z / d \\
a z, c
\end{array} ; q, d / a\right] .
$$

and

$$
\begin{aligned}
& { }_{2} \psi_{2}\left[\begin{array}{l}
a, b \\
c, d
\end{array} ; q, z\right]=\frac{(a z, b z, c q / a b z, d q / a b z ; q)_{\infty}}{(q / a, q / b, c, d ; q)_{\infty}} \times
\end{aligned}
$$

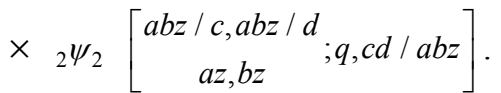

(i) Let $q \rightarrow q^{2}, a, b \rightarrow \infty$, and take $z=q^{2} / a b, \quad c=-q$, $d=-q$, in (7.1) and (7.2), respectively, to get

$$
\left(q ; q^{2}\right)_{\infty} \bar{\psi}_{0, c}(q)=\sum_{n=-\infty}^{\infty} \frac{\left(-q ; q^{2}\right)_{n} q^{n^{2}}}{\left(-q^{2} ; q^{2}\right)_{n}}
$$

and

$$
\begin{aligned}
\bar{\psi}_{0, c}(q) & =\sum_{n=-\infty}^{\infty}\left(-1 ; q^{2}\right)_{n}\left(-q ; q^{2}\right)_{n} q^{n} \\
& =\sum_{n=-\infty}^{\infty}(-1 ; q)_{2 n} q^{n}=2 \sum_{n=-\infty}^{\infty}(-q ; q)_{2 n-1} q^{n} \\
& =2 q \sum_{n=-\infty}^{\infty}(-q ; q)_{2 n+1} q^{n} .
\end{aligned}
$$

(ii) $\quad\left(q ; q^{2}\right)_{\infty} \bar{\psi}_{1, \mathrm{c}}(q)=\sum_{n=-\infty}^{\infty} \frac{q^{n^{2}+2 n}\left(-q ; q^{2}\right)_{n}}{\left(-q^{2} ; q^{2}\right)_{n}}$.

$\left(q \rightarrow q^{2}, a, b \rightarrow \infty, c=-q^{2}, \quad d=-q^{3}, z=q^{4} / a b\right.$ in (7.1))

$$
\bar{\psi}_{1, \mathrm{c}}(q)=2 \sum_{n=-\infty}^{\infty}(-q ; q)_{2 n} q^{n} .
$$

$\left(q \rightarrow q^{2}, a, b \rightarrow \infty, c=-q^{2}, \quad d=-q^{2}, z=q^{4} / a b\right.$ in 7.2) $)$

(iii) $\frac{\mathrm{q}}{(1+\mathrm{q})}\left(q ; q^{2}\right)_{\infty} \bar{\phi}_{0, c}(q)=\sum_{n=-\infty}^{\infty} \frac{q^{n^{2}+3 n}\left(-q^{2} ; q^{2}\right)_{n}}{\left(-q^{3} ; q^{2}\right)_{n}}$.

$$
\left(q \rightarrow q^{2}, \quad a=-q^{2}, b=-q^{3}, c=d=0, \quad z=q\right. \text { in (7.1)) }
$$

$$
\begin{gathered}
\bar{\phi}_{0, c}(q)=\frac{q}{2} \sum_{n=-\infty}^{\infty} \frac{q^{2 n^{2}+4 n}}{(-q ; q)_{2 n+2}}=\frac{1}{2 q} \sum_{n=-\infty}^{\infty} \frac{q^{2 n^{2}}}{(-q ; q)_{2 n}}=\frac{1}{2 q} \bar{\psi}_{0, \mathrm{c}}(q) . \\
\left(q \rightarrow q^{2}, \quad a=-q^{2}, \quad b=-q^{3}, c=d=0, \quad z=q \text { in }(7.2)\right) \\
\text { (iv) } \quad\left(q ; q^{2}\right)_{\infty} \bar{\phi}_{1, c}(q)=\sum_{n=-\infty}^{\infty} \frac{q^{n^{2}+n}\left(-q^{2} ; q^{2}\right)_{n}}{\left(-q ; q^{2}\right)_{n+1}}=T_{1, c}(q) . \\
\left(q \rightarrow q^{2}, \quad a=-q^{2}, b=-q, c=d=0, \quad z=q \text { in }(7.1)\right) \\
\bar{\phi}_{1, c}(q)=\frac{1}{2(1+q)} \sum_{n=-\infty}^{\infty} \frac{q^{2 n^{2}+2 n}}{\left(-q^{2} ; q\right)_{2 n}}=\frac{1}{2} \sum_{n=-\infty}^{\infty} \frac{q^{2 n^{2}+2 n}}{(-q ; q)_{2 n+1}} . \\
\left(q \rightarrow q^{2}, \quad a=-q, b=-q^{2}, c=d=0, \quad z=q \text { in }(7.2)\right)
\end{gathered}
$$

\section{Relationship between Bilateral Mock Theta Functions}

From these alternative definition it is interesting to note that bilateral mock theta functions developed by Andrews can be expressed as bilateral mock theta functions developed by Bringmann et al. 
From (7.3)

$$
\left(q ; q^{2}\right)_{\infty} \bar{\psi}_{0, c}(q)=S_{0, c}(q)
$$

From (7.4)

$$
\bar{\psi}_{0, c}(q)=2 q \bar{\phi}_{0, c}(q)
$$

From (7.5)

$$
\left(q ; q^{2}\right)_{\infty} \bar{\psi}_{1, c}(q)=S_{1, c}(q)
$$

From (7.6)

$$
\bar{\psi}_{1, c}(q)=2 \bar{\phi}_{1, c}(q)
$$

From (7.7)

$$
\frac{q^{3}}{(1+q)^{2}} \bar{\phi}_{0, c}(q)=T_{0, c}(q)
$$

From (7.8)

$$
\bar{\phi}_{0, c}(q)=\frac{1}{2 q} \bar{\psi}_{0, c}(q)
$$

From (7.9)

From (7.10)

$$
\left(q ; q^{2}\right)_{\infty} \bar{\varphi}_{1, c}(q)=T_{1, c}(q)
$$

$$
\bar{\phi}_{1, c}(q)=\frac{1}{2} \bar{\psi}_{1, c}(q) .
$$

$S_{0}(q), S_{1}(q), T_{0}(q)$ and $T_{1}(q)$ are eighth order mock theta functions given by Gordon and McIntosh [8].

\section{Extended Form for Bilateral 'Complete' Mock Theta Functions}

We define the extended 'Complete' mock theta functions:

$$
\begin{gathered}
\bar{\psi}_{0, c}(\alpha, z, q)=\frac{1}{(z)_{\infty}} \sum_{n=-\infty}^{\infty} \frac{(z)_{n} q^{2 n^{2}-n+n \alpha}}{(-q ; q)_{2 n}}, \\
\bar{\psi}_{1, c}(\alpha, z, q)=\frac{1}{(z)_{\infty}} \sum_{n=-\infty}^{\infty} \frac{(z)_{n} q^{2 n^{2}+n+n \alpha}}{(-q ; q)_{2 n+1}} \\
\bar{\phi}_{0, c}(\alpha, z, q)=\frac{1}{(z)_{\infty}} \sum_{n=-\infty}^{\infty}(z)_{n} q^{n \alpha}(-q ; q)_{2 n+1}
\end{gathered}
$$

and

$$
\bar{\phi}_{1, c}(\alpha, z, q)=\frac{1}{(z)_{\infty}} \sum_{n=-\infty}^{\infty}(z)_{n} q^{n \alpha}(-q ; q)_{2 n} .
$$

For $\alpha=1, z=0$ these extended functions reduce to the bilateral mock theta functions.

We show they are $F_{q}$-functions. Before we prove they are $F_{q}$-functions, we define a $F_{q}$-function.

Truesdell[15] calls the functions which satisfy the functional equation

\section{F-functions.}

$$
\frac{\partial}{\partial z} F(z, \alpha)=F(z, \alpha+1)
$$

The $q$-analogue is that the functions which satisfy the functional equation

where

$$
D_{q, z} F(z, \alpha)=F(z, \alpha+1),
$$

$$
z D_{q, z} F(z, \alpha)=F(z, \alpha)-F(z q, \alpha),
$$

are called $F_{q}$-Functions.

By definition

$$
z D_{q, z} \bar{\phi}_{0, c}(\alpha, z, q)=\bar{\phi}_{0, c}(\alpha, z, q)-\bar{\phi}_{0, c}(\alpha, z q, q)
$$

$=\frac{1}{(z)_{\infty}} \sum_{n=-\infty}^{\infty}(z)_{n} q^{n \alpha}(-q ; q)_{2 n+1}-\frac{1}{(z q)_{\infty}} \sum_{n=-\infty}^{\infty}(z q)_{n} q^{n \alpha}(-q ; q)_{2 n+1}$

$$
\begin{gathered}
=\frac{1}{(z)_{\infty}} \sum_{n=-\infty}^{\infty}(z)_{n} q^{n \alpha}(-q ; q)_{2 n+1}\left[1-\left(1-z q^{n}\right)\right] \\
=\frac{z}{(z)_{\infty}} \sum_{n=-\infty}^{\infty}(z)_{n} q^{n(\alpha+1)}(-q ; q)_{2 n+1} \\
=z \bar{\varphi}_{9 c}(\alpha+1, z, q) .
\end{gathered}
$$

Hence

$$
D_{q, z} \bar{\varphi}_{0, c}(\alpha, z, q)=\bar{\varphi}_{0 c}(\alpha+1, z, q) .
$$

Thus $\bar{\phi}_{0, c}(\alpha, z, q)$ is a $F_{q}$-function.

Similarly, all other functions listed in section 9 are $F_{q}$ functions.

\section{Relations between Extended Functions}

Now we give a relationship between these generalized mock theta functions.

(i) $D_{q, z}^{2} \bar{\psi}_{0, c}(\alpha, z, q)=\frac{1}{(z)_{\infty}} \sum_{n=-\infty}^{\infty} \frac{(z)_{n} q^{2 n^{2}+n+n \alpha}}{(-q ; q)_{2 n}}$

$$
\begin{aligned}
& =\frac{1}{(z)_{\infty}} \sum_{n=-\infty}^{\infty} \frac{(z)_{n} q^{2 n^{2}+n(\alpha+1)}}{(-q ; q)_{2 n+1}}\left(1+\mathrm{q}^{2 \mathrm{n}+1}\right) \\
= & \bar{\psi}_{1, c}(\alpha, z, q)+q D_{q, z}^{2} \bar{\psi}_{1, c}(\alpha, z, q) .
\end{aligned}
$$

(ii) $\bar{\phi}_{0, c}(\alpha, z, q)=\frac{1}{(z)_{\infty}} \sum_{n=-\infty}^{\infty}(z)_{n} q^{n \alpha}(-q ; q)_{2 n}\left(1+q^{2 n+1}\right)$

$$
=\bar{\phi}_{1, c}(\alpha, z, q)+q D_{q, z}^{2} \bar{\phi}_{1, c}(\alpha, z, q) \text {. }
$$

\section{Conclusions}

The study of basic bilateral mock theta functions is interesting as it gives alternative forms and close relationship among the mock theta functions. We have expressed them as ${ }_{2} \varphi_{1}$ series and represented them as continued fractions. The same method can be applied for other classical mock theta functions

I have considered these mock theta functions (1.1)--(1.6) in detail finding their properties and expansions etc [12], [13] and [14]. 


\section{REFERENCES}

[1] R.P. Agarwal, Resonance of Ramanujan's Mathematics III, New Age International (P) Ltd. New Delhi (1996).

[2] G. E. Andrews, $q$-orthogonal polynomials, Rogers- Ramanujan identities, and mock theta functions, preprint.

[3] G. E. Andrews, $q$-Series: Their Development.,CBMS regional conference lecture series 66 (1986), Amer Math. Soc. Providence.

[4] G. E. Andrews, Parity in partition identities, Ramanujan Journal (to appear).

[5] G. E. Andrews, D. Hickerson, Ramanujan's "Lost" Notebook VII: The sixth order mock theta functions, $A d v$. in Math. 89 (1991), 60-105.

[6] Youn-Seo Choi, Tenth order mock theta functions in Ramanujan's 'Lost' Notebook, Invent. Math. 136 (1999), 497-569.

[7] G. Gasper and M. Rahman, Basic Hypergeometric Series, Cambridge University Press, Cambridge (1990).

[8] B.Gordon and R.J. McIntosh, Some eighth order mock theta functions, J. London Math. Soc. 62(2) (2000), 321-335
[9] K. Hikami, Transformation formula of the " 2 nd" order mock theta function, Lett. Math. Phys., 75(1) (2006), 93-98.

[10] E. Mortess, On three third order mock theta functions and Hecke-type double sums (preprint).

[11] S. Ramanujan, Collected Papers, Cambridge University Press, 1972, reprinted Chelsea, New York, 1962.

[12] Bhaskar Srivastava, A generalization and study of four new mock theta functions (Communicated).

[13] Bhaskar Srivastava, Some new mock theta functions, accepted in Math. Sci. Research J.

[14] Bhaskar Srivastava, Partial new mock theta functions (Communicated).

[15] C Truesdell, An essay toward a unified theory of special functions, Princeton University Press, Princeton, (1948).

[16] G.N. Watson, The final problem: An account of the mock theta functions, J. London Math. Soc. 11 (1936), 55-80.

[17] G.N. Watson, The Mock Theta FunctionsII, Proc. London Math. Soc. (2) 42 (1937), 272- 304. 